\title{
Estudio in vitro de microfiltración en obturaciones de clase II de resina compuesta condensable
}

\author{
Lois Mastach FJ*, Paz Roca C*, Pazos Sierra R**, Rodríguez-Ponce A***
}

\section{RESUMEN}

Objetivo: El propósito de este estudio es evaluar la microfiltración en cavidades de clase II con márgenes gingivales situados en esmalte, obturadas con resina compuesta Surefil ${ }^{1}$. Material y métodos: 104 cavidades preparadas en dientes humanos extraídos fueron distribuidas al azar en cuatro grupos $(n=26)$ según la técnica de obturación empleada: grupo I, inserción en bloque; grupo II, inserción en bloque con una base de compómero fluido; grupo III, inserción incremental; grupo IV, inserción incremental con una base de compómero fluido. Las muestras fueron almacenadas en agua durante 24 horas, termocicladas 500 veces entre $5^{\circ}$ y $55^{\circ} \mathrm{C}$, sumergidas en una solución de fucsina básica al 0,5\% durante 24 horas, seccionadas longitudinalmente y examinadas para evaluar la microfiltración. Resultados: El grupo I presentó una microfiltración marginal significativamente superior que los grupos II, III y IV. Conclusión: Aunque ninguna de las técnicas de obturación empleadas pudo evitar completamente la microfiltración, tanto la técnica incremental como el uso de Dyract flow como base cavitaria la redujeron significativamente.

Palabras clave: Microfiltración; Resina compuesta condensable; Cavidad clase II; Base cavitaria.

\begin{abstract}
Objective: The purpose of this study was to evaluate microleakage in Class II cavities with gingival margins in enamel, restored with the condensable resin composite Surefil, placed with a bulk or an incremental filling technique and with or without a liner. Method and materials: 104 cavities prepared on extracted human teeth were randomly distributed into four treatment groups $(n=26)$ : group I, bulk filling technique; group II, bulk filling technique with Dyract flow liner; group III, incremental filling technique; group IV, incremental filling technique with Dyract flow liner. Samples were stored in water for 24 hours, thermocycled between $5^{\circ} \mathrm{C}$ and $55^{\circ} \mathrm{C}$ for 500 cycles, stained with $0.5 \%$ basic fuchsin dye for 24 hours, sectioned longitudinally and scored for microleakage. Results: Group I showed significantly more gingival marginal microleakage than did groups II, III and IV. Conclusion: Even though none of the filling techniques could completely prevent microleakage, the incremental technique and the use of Dyract flow as a liner significantly reduced it.
\end{abstract}

Key words: Microleakage; Condensable resin composite; Class II cavity; Liner.

Aceptado para publicación: octubre 2003.

* Profesor Tutor Clínico de Patología y Terapéutica dental. Facultad de Medicina y Odontología de la Universidad de Santiago de Compostela.

** Profesor Asociado de Patología y Terapéutica dental. Facultad de Medicina y Odontología de la Universidad de Santiago de Compostela.

*** Profesor Titular de Patología y Terapéutica dental. Facultad de Medicina y Odontología de la Universidad de Santiago de Compostela.

Lois Mastach FJ, Paz Roca C, Pazos Sierra R, Rodríguez-Ponce A. Estudio in vitro de microfiltración en obturaciones de clase II de resina compuesta condensable. Av. Odontoestomatol 2004; 20-2: 85-94. 


\section{INTRODUCCION}

La amalgama dental ha sido utilizada como principal material de restauración en dientes posteriores durante más de 100 años. Sin embargo, las nuevas demandas de estética y los problemas relacionados con la toxicidad del mercurio han hecho necesario buscar alternativas a este material.

Las resinas compuestas se introdujeron en el mercado en los años 60 y desde entonces se han utilizado con gran éxito para la restauración de dientes del sector anterior. A partir de los años 80 aparecieron resinas específicamente diseñadas para dientes posteriores pero su aceptación ha sido más reticente y polémica. Ello se debe a que los problemas y limitaciones que presentan las resinas compuestas se manifiestan de forma mucho más acusada cuando se utilizan en el sector posterior. En un principio los principales problemas eran: el desgaste por insuficiente resistencia a la abrasión $(1,2)$, la insuficiente resistencia a la fractura (3), la contracción de polimerización (4-6), la microfiltración $(7,8)$, la sensibilidad postoperatoria (9), la caries secundaria (10-12) y la dificultad en su manipulación clínica debido a su viscosidad que impide su condensación. Algunos de estos problemas han sido prácticamente solucionados en los últimos años gracias a la gran mejora que han experimentado las resinas compuestas en sus propiedades físicas. Así, en algunas de ellas se ha reducido el desgaste hasta niveles comparables a los de la amalgama (13-16); sus propiedades mecánicas también han mejorado sensiblemente (17). Sin embargo la contracción de polimerización, con sus secuelas de desadaptación, microfiltración y caries secundaria, sigue siendo la gran dificultad sin resolver. Además, si se trata de una restauración de clase II, el problema todavía se agrava porque la pared gingival de estas cavidades es la zona más crítica, donde los despegamientos y la microfiltración son más frecuentes, especialmente si los márgenes de la caja proximal se sitúan por debajo de la unión amelo-cementaria. La explicación más comúnmente aducida para este hecho es la escasez o ausencia de esmalte y la más débil adhesión a la dentina (8, 18-20).

Por otra parte, la microfiltración puede verse agravada por los cambios de temperatura que se producen en la boca, debido a los diferentes coeficientes de expansión térmica de los tejidos dentales y de las resinas compuestas (21-23).

Con objeto de minimizar los efectos de la contracción de polimerización en las cajas proximales de las obturaciones de clase II, además de las mejoras en los adhesivos dentinarios (24), se han propuesto una gran variedad de técnicas y procedimientos, tales como: diferentes técnicas incrementales de obturación (incrementos horizontales, oblicuos (25) o con una primera capa de composite autopolimerizable (26)), uso de matrices y cuñas transparentes con diferentes técnicas de fotopolimerización (27), colocación de diferentes bases cavitarias o liners en la pared gingival (CVI (18, 28-30), compomeros (31, 32), o composites fluidos (33), en combinación o no con técnicas incrementales), o la introducción de insertos de cerámica en la masa del composite (34). Sin embargo, ninguna de estas técnicas ha conseguido una aceptación universal, debido a sus resultados variables (35-44).

A finales de los años 90 se introdujo en el mercado una nueva generación de composites denominados "condensables" o "densos" y que mediante modificaciones en sus sistemas de relleno inorgánico tratan de conseguir unas propiedades de manipulación lo más parecidas a las de la amalgama (45-49). Su mayor densidad permite su condensación en la cavidad de forma similar a la amalgama, de tal forma que se las puede apretar contra un punto de contacto bruñido y que no se hundan, manteniendo así su forma. Esto tiene una gran importancia clínica porque facilita considerablemente la realización de contactos interproximales adecuados, contribuyendo así a solucionar uno de los problemas de los composites convencionales cuando se emplean en cavidades de clase II: la dificultad en realizar obturaciones morfológica y funcionalmente correctas $(26,50,51)$. Los fabricantes además afirman que a causa de su elevada densidad estos nuevos composites experimentan una menor contracción de polimerización que los convencionales y se benefician de una mayor profundidad de polimerización, permitiendo su colocación en bloque hasta profundidades de $5 \mathrm{~mm}$ (47-49).

El objetivo de este artículo es estudiar in vitro la microfiltración en el margen gingival de cavidades de clase II obturadas con cuatro técnicas diferentes con 
la resina compuesta condensable Surefil ${ }^{\circledR}$ (DENTSPLY DeTrey).

\section{MATERIAL Y MÉTODOS}

52 molares y premolares humanos extraídos por diferentes circunstancias fueron seleccionados para este estudio. Después de su extracción se limpiaron y fueron conservados en una solución de suero salino hasta su utilización.

Los dientes fueron seleccionados con arreglo a los siguientes criterios: no presentar caries ni descalcificaciones detectables en el área a restaurar; tener la corona íntegra, sin fracturas ni daños por las extracciones y sin restauraciones ni caries, o solamente con caries incipientes, que en ningún caso penetraran hasta el límite amelodentinario. Los dientes fueron aleatoriamente divididos en 4 grupos de 13 .

\section{Preparación cavitaria}

Se prepararon dos cavidades de clase II independientes en cada diente con una fresa 330 de carburo de tungsteno en una turbina de alta velocidad y con refrigeración con aire y agua. Se empleo una fresa nueva después de cada 6 preparaciones. Las dimensiones de las cavidades fueron estandarizadas con arreglo a las siguientes medidas: $4 \mathrm{~mm}$ de anchura vestíbulo-lingual, $4 \mathrm{~mm}$ de altura oclusogingival y $2 \mathrm{~mm}$ de profundidad desde el margen gingival a la pared axial. El margen cavosuperficial gingival de la preparación se situó siempre en el esmalte.

\section{Procedimientos restauradores}

Primeramente, se colocó la matriz de metálica de 7 $\mathrm{mm}$ de ancho y 0,03 $\mathrm{mm}$ de espesor. Seguidamente, las cavidades se grabaron completamente con gel ácido ortofosfórico al $37 \%$ durante 15 segundos, se lavaron durante otros 15 segundos y se secaron para eliminar el exceso de agua, dejando la dentina visiblemente húmeda. Seguidamente se aplicó el adhesivo monocomponente Prime $\varepsilon$ Bond $\mathrm{NT}^{\circledR}$
(DENTSPLY DeTrey) durante 30 segundos, se secó suavemente para eliminar el exceso de disolvente y se polimerizó durante 20 segundos con una lámpara de luz visible Optilux 400 (Demetrón/Kerr). Los dientes, previamente divididos de forma aleatoria, fueron obturados con la resina compuesta Surefil de color B, con cuatro técnicas diferentes:

\section{Grupo I: inserción en bloque}

La resina compuesta condensable Surefil fue colocada directamente en cada cavidad en un solo bloque, condensada y polimerizada desde la superficie oclusal durante 40 segundos y, tras retirar la matriz metálica, durante otros 40 segundos por cada superficie vestibular y lingual.

\section{Grupo II: inserción en bloque con una base de compómero fluido}

Se aplicó una capa horizontal del compómero fluido Dyract flow (DENTSPLY DeTrey) de aproximadamente $1 \mathrm{~mm}$ a $1,5 \mathrm{~mm}$ de espesor sobre la pared gingival, permitiendo que se extendiera hasta el margen cavosuperficial gingival. Se polimerizó desde oclusal durante 20 segundos. Seguidamente, se colocó la resina compuesta condensable Surefil en una única capa, condensándola hasta rellenar toda la cavidad y se polimerizó desde la superficie oclusal durante 40 segundos y, tras retirar la matriz metálica, durante otros 40 segundos por cada superficie vestibular y lingual.

\section{Grupo III: técnica incremental}

La resina compuesta condensable Surefil fue colocada en tres incrementos de forma oblicua: el primero condensado contra las paredes gingival y vestibular ocupando el espacio entre el ángulo gíngivolingual y la pared vestibular hasta aproximadamente $2 / 3$ de su altura; un segundo incremento se condensó también oblicuamente contra la pared lingual; finalmente, un último incremento rellenó el espacio restante de la cavidad hasta la superficie oclusal. Cada incremento se polimerizó durante 40 segundos desde oclusal y, una vez polimerizado el último y reti- 
rada la matriz metálica, otros 40 segundos desde cada superficie vestibular y lingual.

\section{Grupo IV: técnica incremental con una base de compómero fluido}

Se aplicó una capa horizontal del compómero fluido Dyract flow de aproximadamente $1 \mathrm{~mm}$ a $1,5 \mathrm{~mm}$ de espesor sobre la pared gingival, permitiendo que se extendiera hasta el margen cavosuperficial gingival. Se polimerizó desde oclusal durante 20 segundos. Finalmente, se colocó la resina compuesta condensable Surefil en tres incrementos oblicuos siguiendo exactamente la misma técnica aplicada en el grupo II.

\section{Termociclado y tinción de las muestras}

Los dientes se conservaron en agua a temperatura ambiente durante 24 horas y posteriormente fueron sometidos a un proceso de termociclado de 500 ciclos, en agua entre temperaturas de $5^{\circ} \mathrm{C}$ y $55^{\circ} \mathrm{C}$. Los dientes permanecieron en cada baño de agua 30 segundos, con un tiempo de transferencia entre cada baño de otros 30 segundos.

Los ápices radiculares se sellaron con Dyract flow y seguidamente se barnizaron todas las superficies del diente con dos capas de barniz de uñas, hasta una distancia aproximada de $1 \mathrm{~mm}$ por fuera del margen cavosuperficial de las preparaciones.

Los dientes se sumergieron entonces una solución colorante de fucsina básica al $0,5 \%$ durante 24 horas y a temperatura ambiente. Posteriormente se lavaron en agua corriente, se secaron y se cortaron longitudinalmente con un disco de diamante (Komet ${ }^{-}$ 918B/220), en dirección mesio-distal, pasando aproximadamente por el centro de las restauraciones. Las dos secciones obtenidas así fueron examinadas en una lupa estereoscópica con una magnificación de 20X y puntuadas por dos observadores calibrados.

La microfiltración gingival se valoró con arreglo a la siguiente escala:

$-0=\sin$ filtración

- 1 = filtración hasta la mitad de la pared gingival como máximo
- 2 = filtración entre la mitad de la pared gingival y la pared axial, pero sin afectar a esta última

- 3 = filtración a lo largo de la pared axial

Se estudió también la microfiltración desde el margen oclusal con arreglo a la siguiente escala:

$-0=\sin$ filtración

-1 = filtración hasta la mitad de la pared axial como máximo

-2 filtración de más de la mitad de la pared axial

Se examinaron las dos secciones de cada restauración y se acepto como puntuación de la misma aquella que presentó un valor más alto de filtración.

\section{Métodos estadísticos}

Las puntuaciones de microfiltración de cada grupo se analizaron utilizando el test no paramétrico de Kruskal-Wallis con la corrección de Bonferroni para las comparaciones múltiples entre grupos y con un nivel de significación estadística establecido en $\mathrm{p}<0,05$.

\section{RESULTADOS}

Se estudiaron 104 restauraciones en total, divididas en 4 grupos de 26 .

En la tabla 1 se observan las frecuencias de los diferentes grados de microfiltración gingival para cada uno de los grupos estudiados. Ninguno de ellos se vio completamente libre de microfiltración pero, los peores resultados se encuentran en el grupo I (Surefil en bloque) en el que solamente está libre de microfiltración gingival el $19.2 \%$ de las preparaciones, frente a los otros tres grupos, que presentan porcentajes que se sitúan entre el $65.4 \%$ del grupo III (Surefil en bloque/Dyract flow) y el $76.9 \%$ del grupo IV (Surefil en capas/Dyract flow), que es el que presenta mejores resultados. El grupo I presenta también una frecuencia claramente superior de microfiltración severa (grado 3) que los otros tres grupos. Estas diferencias entre el grupo I y los otros tres son estadísticamente significativas $(\mathrm{p}<0.05)$. 
TABLA 1. FRECUENCIAS DE LOS DIFERENTES GRADOS DE MICROFILTRACIÓN GINGIVAL

\begin{tabular}{|c|c|c|c|c|c|c|c|c|}
\hline \multirow[t]{3}{*}{ ‘Grupo } & \multicolumn{8}{|c|}{ Grado de microfiltración } \\
\hline & \multicolumn{2}{|c|}{0} & \multicolumn{2}{|c|}{1} & \multicolumn{2}{|c|}{2} & \multicolumn{2}{|c|}{3} \\
\hline & $N$ & $\%$ & $N$ & $\%$ & $n$ & $\%$ & $n$ & $\%$ \\
\hline I. Surefil en bloque & 5 & 19,2 & 9 & 34,6 & 6 & 23,1 & 6 & 23,1 \\
\hline II. Surefil en bloque/Dyract flow & 18 & 69,2 & 5 & 19,2 & 2 & 7,7 & 1 & 3,8 \\
\hline III. Surefil en capas & 17 & 65,4 & 3 & 11,5 & 3 & 11,5 & 3 & 11,5 \\
\hline IV. Surefil en capas/Dyract flow & 20 & 76,9 & 4 & 15,4 & 2 & 7,7 & 0 & 0,0 \\
\hline
\end{tabular}

\section{TABLA 2. FRECUENCIAS DE LOS DIFERENTES GRADOS DE MICROFILTRACIÓN OCLUSAL}

\begin{tabular}{|c|c|c|c|c|c|c|}
\hline \multirow[t]{3}{*}{ ‘Grupo } & \multicolumn{6}{|c|}{ Grado de microfiltración } \\
\hline & \multicolumn{2}{|c|}{0} & \multicolumn{2}{|c|}{1} & \multicolumn{2}{|c|}{2} \\
\hline & $n$ & $\%$ & $N$ & $\%$ & $n$ & $\%$ \\
\hline I. Surefil en bloque & 26 & 100,0 & 0 & 0,0 & 0 & 0,0 \\
\hline II. Surefil en bloque/Dyract flow & 25 & 96,2 & 1 & 3,8 & 0 & 0,0 \\
\hline III. Surefil en capas & 23 & 88,5 & 2 & 7,7 & 1 & 3,8 \\
\hline IV. Surefil en capas/Dyract flow & 24 & 92,3 & 2 & 7,7 & 0 & 0,0 \\
\hline
\end{tabular}

Los resultados en lo que se refiere a la microfiltración en el margen oclusal se muestran en la tabla 2. No se encontraron diferencias estadísticamente significativas entre ninguno de los cuatro grupos estudiados.

La microfiltración en el margen gingival fue claramente superior en comparación con la registrada en el margen oclusal, tanto para el conjunto de la muestra, como para cada uno de los grupos de estudio (Tablas 1 y 2 )

\section{DISCUSIÓN}

Ninguna de las cuatro diferentes técnicas de obturación empleadas en este estudio consiguió garantizar un sellado marginal completo en la pared gingival de las obturaciones, todas presentaron en alguna proporción microfiltración entre moderada y severa. Los márgenes de las preparaciones se situaron en el esmalte y no es excesivamente aventurado suponer que si se hubieran situado en el cemento los resultados habrían sido peores porque este es un hecho sólidamente corroborado por la bibliografía (8, 18$20,52,53)$. Esto demuestra que la pared gingival de las restauraciones de clase II sigue siendo un problema en las obturaciones de resinas compuestas, y ello a pesar de las mejores propiedades que, según los fabricantes, presentan las resinas condensables. Sin embargo, estudios recientes no han encontrado mejoras significativas en las propiedades físicas de los composites condensables respecto a las de los composites híbridos convencionales. Así, tanto Cobb et al (54) como Kelsey et al (55) y Choi et al (56) en sus respectivos trabajos encuentran que los composites condensables estudiados tienen propiedades mecánicas comparables, y en algún caso inferiores, a las de los híbridos convencionales. En lo que se refiere a la contracción de polimerización, Aw y Nicholls (57) encontraron que solamente Alert ${ }^{\text {to }}$ y $\mathrm{P}^{\circ}$ demostraron significativamente menor contracción que los demás, mientras que los otros cua- 
tro condensables estudiados, entre ellos Surefil, no diferían significativamente de los híbridos convencionales. Según Choi et al (56) estos resultados serían previsibles teniendo en cuenta la naturaleza química similar de las resinas orgánicas de los condensables y de los composites convencionales.

El hallazgo más importante del presente estudio es el significativamente peor resultado en cuanto a microfiltración del grupo obturado con la técnica de inserción en bloque sin base cavitaria, en el que solamente el $19,2 \%$ de las preparaciones consiguieron un sellado marginal completo en la pared gingival frente al $65,4 \%$ del grupo obturado incrementalmente. El uso de técnicas incrementales en la obturación con resinas compuestas goza de amplia aceptación y numerosos estudios apoyan su utilización afirmando que reducen las tensiones derivadas de la contracción de polimerización y mejoran el sellado marginal $(26,27,38-40)$. Su justificación teórica se basa en que los incrementos parciales tienen un factor $\mathrm{C}$ (proporción entre superficies adheridas y no adheridas de una obturación) más favorable y que un menor volumen de material experimentará una menor contracción (58); de esta forma se transmitirían menores tensiones a las superficies adheridas y la contracción de polimerización se produciría en mayor medida a costa de las superficies no adheridas. En un artículo publicado en 1998, Versluis et al (59) estudian el proceso de polimerización de las resinas compuestas simulándolo en el ordenador mediante un modelo matemático que denominan y llegan a la conclusión de que el composite no se contrae hacia la luz, tal como es comúnmente aceptado, sino que la dirección en que lo hace está predominantemente determinada por la forma de la cavidad y la calidad de la adhesión. De acuerdo con esta teoría, si se consigue una buena adhesión a las paredes de la cavidad la contracción de polimerización se producirá fundamentalmente a expensas de las superficies libres, y esto se producirá en mayor medida cuanto más favorable sea el factor $\mathrm{C}$ e independientemente de la posición de la luz.

Sin embargo, otros estudios no han encontrado ninguna ventaja con las técnicas incrementales de obturación. Así, Gallo, Bates E Burgess (37) en un estudio realizado con tres composites condensables no encontraron diferencias estadísticamente significati- vas en cuanto a microfiltración y adaptación marginal entre la obturación incremental o en bloque.

Versluis et al (35) en 1996 estudiaron las tensiones producidas por la contracción de polimerización de diferentes técnicas de obturación mediante una simulación matemática, y concluyeron que las técnicas incrementales provocaban mayores tensiones porque aumentaban la deformación del diente. Sin embargo, reconocen que las técnicas incrementales podrían justificarse por otras razones como la adaptación, profundidad de polimerización y el establecimiento de una adecuada adhesión al diente. Así, Choi et al (56) en su estudio sobre las propiedades de cinco composites condensables, entre ellos Surefil, afirman que no se logra una adecuada polimerización con espesores de material superiores a $2 \mathrm{~mm}$.

El proceso de contracción de polimerización de las resinas compuestas cuando están adheridas a las paredes de una cavidad dista mucho de ser comprendido en su totalidad. Sin embargo, aunque las razones teóricas que lo expliquen no estén muy claras, los resultados experimentales de este estudio apoyan el uso de las técnicas incrementales de obturación.

La utilización de Dyract flow como base cavitaria bajo el composite condensable produjo unos resultados significativamente mejores en cuanto a microfiltración. Mientras que en el grupo I (inserción en bloque) solamente el 19,2\% de las preparaciones estaban libres de microfiltración, en el grupo II (inserción en bloque con Dyract flow) lo estaban el 69,2\%. En los dos grupos obturados incrementalmente (III y IV), el uso de Dyract flow también produjo mejores resultados, pero la diferencia no fue estadísticamente significativa.

Algunos estudios no han encontrado que la utilización de estas bases cavitarias bajo las obturaciones de resina compuesta disminuyera la microfiltración (42-44). Sin embargo, su uso es apoyado por otros muchos trabajos, tanto si se trata de CVI (18, 28-30), de compómeros $(31,32)$, o de composites fluidos $(33,60)$. Tung et al (60) estudiaron la microfiltración de cavidades de clase II restauradas con Surefil insertado en bloque, y obtuvieron resultados significativamente mejores en el grupo en que emplearon una 
base cavitaria de composite fluido. La explicación que estos autores aducen es que la mayor flexibilidad del composite fluido le permitiría actuar como un amortiguador de las tensiones derivadas de la contracción de polimerización. Esta idea está también apoyada por Kemp-Scholte y Davidson (61) y por Van Meerbeek et al (62) y es aplicable a cualquier material utilizado como base cavitaria que tenga un bajo módulo de elasticidad.

En principio, los materiales que parecen más adecuados para su utilización como bases bajo las restauraciones son los composites y compómeros fluidos, por sus mejores propiedades físicas, aunque sus resultados a largo plazo requieren estudios clínicos in vivo.

\section{CONCLUSIONES}

- Ninguna de las técnicas de obturación estudiadas consiguió evitar completamente la microfiltración en la pared gingival de las obturaciones.

- La técnica de obturación en bloque sin base cavitaria presentó significativamente la mayor microfiltración.

- Tanto la técnica de inserción incremental, como la utilización de una base cavitaria de compómero fluido, redujeron significativamente la microfiltración.

\section{BIBLIOGRAFÍA}

1. Leinfelder KF. Wear patterns and rates of posterior composite resins. Int Dent J 1987; 37 : 152-7.

2. Swift EJ Jr. Wear of composite resins in permanent posterior teeth. J Am Dent Assoc 1987; 115: 584-8.

3. Leinfelder KF. Current developments in posterior composite resins. Advanced Dental Research 1988; 2: 115-21.
4. Walls AWG, McCabe JF, Murray JJ. The polymerization contraction of visible-light activated composite resin. J Dent 1988; 16: 177-81.

5. De Gee AJ, Davidson CL, Smith A. A modified dilatomer for continous recording of volumetric polymerization shrinkage of composite restorative materials. J Dent 1981; 9(1): 36-42.

6. Bausch JR, De Lange K, Davidson CL, Peters A, de Gee AJ. Clinical significance of polymerization shrinkage of composite resins. J Prosthet Dent 1982; 48: 59-67.

7. Eakle WS, Ito RK. Effect of insertion technique on microleakage in mesio-occlusodistal composite resin restorations. Quintessence Int 1990; 21: 369-374.

8. Cvitko E, Denehy GE, Boyer DB. Effect of matrix systems and polymerization technique on microleakage of class II composite restorations. Am J Dent 1992; 5:321-323.

9. Eick JD, Welch FH. Polymerization shrinkage of posterior composite resins and its possible influence on postoperative sensitivity. Quintessence International 1986; 17: 103-11.

10. Köhler B, Rasmusson C-G, Ödman P. A five-year clinical evaluation of class II composite resin restorations. J Dent 2000; 28: 111-16.

11. Barnes DM, Blank LW, Thompson VP, Holston AM, Gingel JC. A 5- and 8-year clinical evaluation of a posterior composite resin. Quintessence Int 1991; 22 (2): 143-51

12. Mjör IA. The location of clinically diagnosed secondary caries. Quintessence Int 1998; 29: 313-16.

13. Johnson GH, Bales DJ, Gordon GE, Powell LV. Clinical performance of posterior composite restorations. Quintessence International 1992; 23: 705-11.

14. Willems G, Lambrechts P, Braem M, Vanherle G. Three year follow-up of five posterior composites: In vivo wear results. J Dent 1993; 21: 74-8. 
15. Leinfelder KF. Posterior composites. State-ofthe-art clinical applications. Dent Clin North Am 1993; 37: 411-8.

16. Taylor DF, Bayne SC, Leinfelder KF, Davis S, Koch GG. Pooling of long-term clinical wear data for posterior composites. Am J Dent 1994; 7: 167-74.

17. Lutz F. State of the art of tooth-colored restoratives. Oper Dent 1996; 21: 237-248

18. Crim GA, Chapman KW. Reducing microleakage in class II restorations: An in vitro study. Quitessence Int 1994; 25: 781-785.

19. Schuckar M, Geurtsen W. Proximo-cervical adaptation of class II-composite restoration after thermocycling: A quantitative and qualitative study. J Oral Rehabil 1997; 24: 766-775.

20. Ferrari M, Davidson CL. Sealing performence of Scotchbond Multi-purpose-Z100 in class II restorations. Am J Dent 1996; 9 (4): 145-9.

21. Bullard RH, Leinfelder KF, Russell CM. Effect of coefficient of thermal expansion on microleakage. J Am Dent Assoc 1988; 116: 871-874.

22. Momoi $H$, Iwase $H$, Nakano $Y$, Kohno A, Asanuma A, Yanagisawa K. Gradual increase in marginal leakage of resin composite restorations. J Dent Res 1990; 69: 1659-1663.

23. Versluis A Douglas WH, Sakaguchi RL. Thermal expansion coefficient of dental composites measured with strain gauges. Dent Mater 1996; 12(5): $290-4$

24. Wakefield CW, Draughn RA, Sneed WD, Davis TN. Shear bond strengths of six bonding systems using the pushout method of in vitro testing. Oper Dent 1998; 23: 69-76.

25. Pollack BF. Class II composite. Oral Health 1988; 78: 23-5.

26. Bertolotti RL. Posterior composite technique utilizing directed polymerization shrinkage and a novel matrix. Pract Periodontics Aesthet Dent 1991; 3 (4): 53-8.

27. Lutz F, Krejci I, Barbakow F. Quality and durability of marginal adaptation in bonded composite restorations. Dent Mater 1991; 7(2): 107-13.

28. Abuoshala A, Kugel G, Hurley E. Class II composite resin restorations using glass-ionomer liners: Microleakage studies. J Clin Ped Dent 1996; 21: 67-71.

29. Prati C. Early marginal microleakage in class II resin composite restorations. Dent Mater 1989; 5 (6): 392-8.

30. Wibowo G, Stockton L. Microleakage of Class II Composite restorations. Am J Dent 2001; 14: 177-85.

31. Tolidis K, Nobecourt A, Randall RC. Effect of a resin-modified glass ionomer liner on volumetric polymerization shrinkage of various composites. Dent Mater 1998; 14(6): 417-23.

32. Dietrich T, Kraemer M, Losche GM, Werncke Kd, Roulet JF. Influence of dentin conditioning and contamination on the marginal integrity of sandwich Class II restorations. Oper Dent 2000; 25: 401-10.

33. Leevailoj C, Cochran MA, Matis BA, Moore BK, Platt JA. Microleakage of posterior packable resin composites with and without flowable liners. Oper Dent 2001; 26: 302-7.

34. Rada ER. Class II direct composite resin restorations with beta-quartz glass-ceramic inserts. Quintessence Int 1993; 24: 793-98

35. Versluis A, Douglas WH, Cross M, Sakaguchi RL. Does and incremental filling technique reduce polymerization shrinkage stress? J Dent Res 1996; 75: 871-8.

36. Tjan $\mathrm{AH}$, Berg $\mathrm{BH}$, Linder $\mathrm{C}$. Effect of various incremental techniques on the marginal adaptation of class II composite resin restorations. J Prosthet Dent 1992; 67: 62-6. 
37. Gallo JR 3rd, Bates ML, Burgess JO. Microleakage and adaptation of class II packable resin-based composites using incremental or bulk filling techniques. Am J Dent 2000; 13 (4): 205-8.

38. De Wet FA, Exner HV, du Preez, van Niekerk JP. The effect of placement technique on marginal adaptation of posterior resins. J Dent Assoc S Afr 1991 ; 46 (3): 171-4.

39. Wilson EG, Mandradjieff $M$, Brindock $T$. Controversies in posterior composite resin restorations. Dent Clin North Am 1990; 34(1): 27-44.

40. Torstenson B, Oden A. Effects of bonding agent types and incremental techniques on minimizing contraction gaps around resin composites. Dent Mater 1989; 5(4): 218-23.

41. Opdam NJ, Roeters JJ, Burgersdijk RC. Microleakage of class II box-type composite restorations. Am J Dent 1998; 11 (4): 160-4.

42. Haller B, Trojanski A. Effect of multi-step dentin bonding systems and resin-modified glass ionomer cement liner on marginal quality of dentinbonded resin composite restorations. Clin Oral Investig 1998; 2 (3): 130-6

43. Chuang SF, Liu JK, Chao CC, Liao FP, Chen YH. Effects of flowable composite lining and operator experience on microleakage and internal voids in class II composite restorations. J Prosthet Dent 2001; 85: 177-83.

44. Miranda WG Jr, Nunes MF, Cardoso PE, Santos JF. Microleakage of condensable composite resins combined with a flowable composite (abstract 1603). J Dent Res 1999; 78 (special issue): 306.

45. Leinfelder K, Prasad A. A new condensable composite for the restoration of posterior teeth. Dent Today 1998; 17: 112-116.

46. Leinfelder K. A report on a new condensable composite resin. Compend Contin Educ Dent 1998; 19: 220-238.
47. Surefil technical manual. Milford, Del.: DENTSPLY/L.D. Caulk; 1998: 1-23.

48. Prodigy technical manual. Orange, Calif.: SDS Kerr; 1998: 1-18

49. Filtek P-60 technical manual. Minneapolis: $3 M$ Dental Products; 1999: 5-33

50. Baratieri LN, Ritter AV, Perdigao J, Felippe LA. Direct posterior composite resin restorations: current concepts for their technique. Pract Periodontics Aesthet Dent 1998; 10: 875-86.

51. Small BW. Direct posterior composite restorations: state of the art in 1998. J Gen Dent 1998; 46: 26-32.

52. Beznos C. Microleakage at the cervical margin of composite class II cavities with different restorative techniques. Oper Dent 2001; 26: 60-9.

53. Meiers JC, Kazemi R, Meier CD. Microleakage of packable composite resins. Oper Dent 2001; 26 : 121-6.

54. Cobb DS, MacGregor KM, Vargas MA, Denehy GE. The physical properties of packable and conventional posterior resin-based composites: a comparison. J Am Dent Assoc 2000; 13 (11): 1610-5.

55. Kelsey WP, Latta MA, Shaddy RS, Stanislav CM. Physical properties of three packable resin-composite restorative materials. Oper Dent 2000; 25: $331-5$.

56. Choi KK, Ferracane JL, Hilton TJ, Charlton D. Properties of packable dental composites. J Esthet Dent 2000; 12: 216-26.

57. Aw TC, Nichols JI. Polymerization shrinkage of densely-filled resin composites. Oper Dent 2001; 26: 498-504.

58. McCullock AJ, Smith BGN. In vitro studies of cuspal movement produced by adhesive restorative materials. Br Dent J 1986; 161: 405-9. 
59. Versluis A, Tantbirojn D, Douglas WH. Do dental composites always shrink toward the light?. J Dent Res 1998; 77: 1435-1445.

60. Tung FF, Estafan D, Scherer W. Microleakage of a condensable resin composite: An in vitro investigation. Quintessence Int 2000; 31: 430-434.

61. Kemp-Scholte CM, Davidson CL. Complete marginal seal of Class V resin composite restorations effected by increased flexibility. J Dent Res 1990; 69(6):1240-3.
62. Van Meerbeek B, Willems G, Celis JP, Roos JR, Braem M, Lambrechts P, Vanherle G. Assessment by nano-indentation of the hardness and elasticity of the resin-dentin bonding area. Dent Res 1993; 72(10): 1434-42.

\section{CORRESPONDENCIA}

Francisco Javier Lois Mastach. Avda Juan XXIII n ${ }^{\circ}$, 15704 Santiago de Compostela.

Correo electrónico: FJLOIS@ terra.es 